\title{
November 23 Highlights
}

\section{Interferon beta for secondary progressive MS}

Although several studies have demonstrated benefits of interferon beta in relapsing-remitting MS, effects in other forms of the disease have not been fully examined. Panitch et al. report the North American study of interferon beta- $1 \mathrm{~b}$ in secondary progressive MS, which showed no effect of treatment on the primary endpoint-progression of disability. However, treatment significantly decreased relapses, active MRI lesions, and accumulation of disease burden of MRIs.

see page 1788

Kappos et al. examined possible reasons why these results differed from another large controlled study of the same agent and nearly identical design. In a combined analysis they found an overall risk reduction for confirmed disease progression by about $20 \%$ and explored characteristics that would allow identifying responders. At least one relapse and change in EDSS by $>1$ point in the 2 years prior to study entry were the only predictors of a positive treatment response that were consistently identified in both studies.

see page 1779

In the accompanying editorial, Cohen and Antel discuss the implications of these studies. These include well-known cautions against comparing across studies and new hypotheses about the role of inflammatory and degenerative processes in MS progression.

see page 1768

\section{IMPA2 haplotype associated with febrile seizures}

Nakayama et al. identified a novel febrile seizure (FS) locus on chromosome 18p11.2 in a genome-wide linkage screen of 48 Japanese nuclear families with FS. Subsequent genetic association analysis suggested that variants in IMPA2 (myo-inositol monophosphatase 2), a gene in the 18p linkage region, may confer susceptibility to FS.

see page 1803

In the accompanying editorial, Winawer and Hesdorffer review the epidemiology of FS and evidence for a genetic contribution to FS susceptibility. They describe the five chromosomal regions implicated by genetic linkage studies to harbor FS susceptibility genes (FEB1-FEB5), as well as genes responsible for syndromes with heterogenous phenotypes that include FS, such as GEFS+ and SMEl. Most genes implicated in human idiopathic seizure disorders have been ion channel and receptor genes. The IMPA2 candidate suggests a novel mechanism for seizure susceptibility.

see page 1770

\section{Brain reorganization from cortical malformations}

A magnetoencephalographic study by Burneo et al. found evidence of brain reorganization with respect to malformations of cortical development. Reorganization was specific to dysplastic and not simple migration disturbances unless the malformation involved large areas of absent cortical development. In these cases, complete transhemispheric reorganization was evident.

see page 1818

\section{Magnetoencephalography (MEG): Reorganization of language-specific cortex}

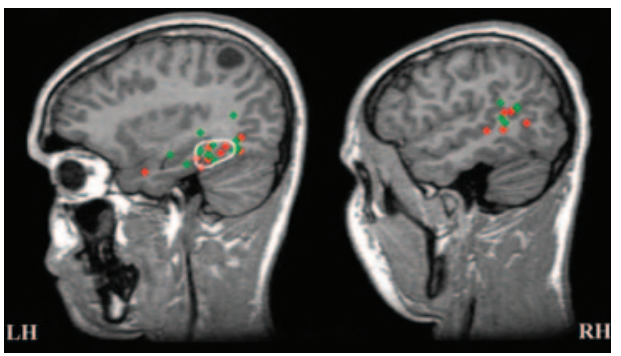

Activation profile in a patient with a pilocytic astrocytoma in the left posterior parietal area. Atypical localization of receptive language in the left hemisphere.

Pataraia et al. examined brain activation profiles for receptive language function in 21 patients with left hemisphere lesions and 23 patients with left mesial temporal lobe epilepsy (MTS). Results indicated an increased probability for interhemispheric reorganization of the brain mechanism for receptive language function in MTS patients. Space-occupying lesions were primarily associated with atypical location of the languagespecific cortex within the dominant (left) hemisphere.

see page 1825

The editorial by Shih and Cohen that accompanies these articles notes that it is firmly established that the adult CNS is capable of adaptive changes and substantial cortical reorganization leading to recovery of function. Mechanisms include dendritic growth, synaptogenesis, changes in synaptic efficiency (i.e., long-term potentiation or depression), and reorganizational changes in body part representations. Cortical plasticity can be enhanced by interventions such as training protocols, pharmacologic interventions, and possibly brain stimulation. MEG can detect an area of cortical activation and accurately track the subsequent activation of functionally connected cortical regions over time. However, before one ascribes too much significance to MEG activation maps, we need to better understand the specific functional role of activated regions, their contribution to task performance or function recovery, and their significance in terms of the activity (excitatory vs inhibitory).

see page 1772 
- Reversible lesions in the splenium

Tada et al. reported 15 patients with a reversible lesion in the splenium of the corpus callosum. Homogenously reduced diffusion was observed in 7/7 patients who had DWI. The clinical features were similar: altered consciousness in all with seizures in at least 7 patients. Complete clinical recovery occurred within a month and the MRI lesion resolved.

see page 1854

\section{Cholesterol associated stroke risk varies in subgroups}

This case-control study by Tirschwell and N.L. Smith found that higher total and lower HDL cholesterol levels were associated with increased risk of ischemic stroke, especially for large artery and lacunar subtypes. These associations appear more important in young patients, those with low HDL, non-diabetics, and those without atrial fibrillation. The lowest levels of total cholesterol were associated with an increased risk of hemorrhagic stroke.

see page 1868

\section{Being overweight and brain atrophy}

Gustafson et al. provide further evidence that being overweight through mid- and late-life is not good for brain health in women. Higher body mass index was shown to be related to the occurrence of temporal lobe atrophy over a 32-year follow-up period.

see page 1876

\section{Blood patch in spontaneous intracranial hypotension}

Berroir et al. showed that spontaneous intracranial hypotension with typical orthostatic headache can be diagnosed without performing lumbar puncture or identifying a cerebrospinal fluid leak and can be cured by early epidural blood patch in $77 \%$ of patients.

see page 1950

\section{Sirolimus: Less neurotoxicity}

The calcineurin inhibitors cyclosporin and tacrolimus often have major neurotoxicity. Maramattom and Wijdicks reviewed over 200 transplant recipients treated with sirolimus. Neurotoxicity was not encountered.

see page 1958

\section{Cortical myoclonus and $M E C P 2$ gene}

Leuzzi et al. provide evidence for the cortical origin of myoclonus in a male with $M E C P 2$ gene alteration. The lack of cortical hyperexcitability suggests a severe derangement of transcortical synaptic connectivity.

see page 1968

\section{Frequency-specific mal de debarquement}

Richard F. Lewis found that mal de debarquement-a feeling of persistent movement that follows passive motion - can reflect aberrant adaptation depending on the dynamics of the preceding movement.

see page 1983 


\section{Neurology}

\section{November 23 Highlights}

Neurology 2004;63;1766-1767

DOI 10.1212/WNL.63.10.1766

This information is current as of November 22, 2004

\section{Updated Information \&}

Services

Permissions \& Licensing

Reprints including high resolution figures, can be found at: http://n.neurology.org/content/63/10/1766.full

Information about reproducing this article in parts (figures,tables) or in its entirety can be found online at:

http://www.neurology.org/about/about_the_journal\#permissions

Information about ordering reprints can be found online:

http://n.neurology.org/subscribers/advertise

Neurology ${ }^{\circledR}$ is the official journal of the American Academy of Neurology. Published continuously since 1951, it is now a weekly with 48 issues per year. Copyright . All rights reserved. Print ISSN: 0028-3878. Online ISSN: 1526-632X.

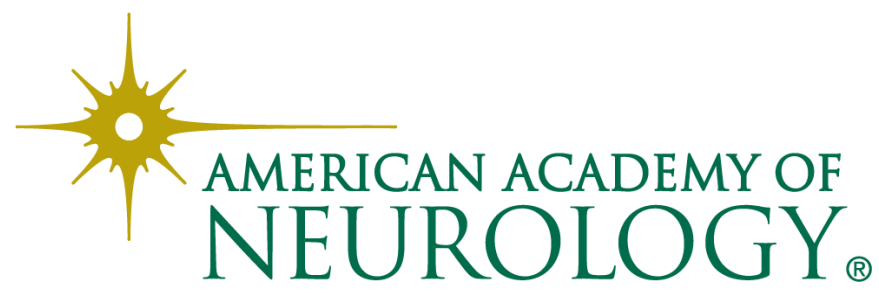

